\title{
Determining the Feasibility of Forearm Mounted Vibrotactile Displays
}

\author{
Ian Oakley*, Yeongmi Kim ${ }^{\dagger}$, Junhun Lee ${ }^{\ddagger} \&$ Jeha Ryu** \\ HMCI Lab, Dept of Mechatronics, Gwangju Institute of Science and Technology
}

\begin{abstract}
At first glance, multi-element forearm mounted vibrotactile displays would appear to have considerable potential as an output device for mobile computing. The devices are small, robust and discrete, and the body site both easily accessible and socially acceptable for such a purpose. However, due to the absence of a thorough account of vibrotactile perception, it is hard to determine their feasibility, or even what might form an appropriate arrangement of vibrating elements or tactors. We describe two studies intended to shed light on these issues. The first extends the localization literature relating to the forearm, and its results indicate that different spatial arrangements of tactors can result in substantially different levels of performance. The second study examines the influence of adjusting the size of the area of the skin experiencing a vibration with its perceived intensity. The results indicate a positive relationship between increased size and increased perceived intensity. Finally, the implications of these studies for the design of vibrotactile arrays are discussed.

CR Categories and Subject Descriptors: H.5.2 [User Interfaces]: Haptic Interfaces, H.1.2 [User/Machine Systems]: Human Factors, I.3.6 [Methodology and Techniques]: Interaction techniques.
\end{abstract}

Additional Keywords: Vibrotactile, psychophysics

\section{INTRODUCTION}

It has long been accepted that the skin, the largest organ in the body, has considerable potential as a conduit for information. Correspondingly, there is a substantial history of research investigating how it might be effectively utilized. This effort has led to the development of a wide variety of skin stimulation technologies, ranging from electrical to vibratory to pneumatic. Of these technologies, large scale vibrotactile displays, utilizing motor or transducer based vibrating elements, and considered either individually or in groups, have arguably been the most popular. The reasons for this are straightforward and easy to enumerate: they are simple to construct, small, cheap, robust, reliable, and consume modest amounts of power.

Initial investigations with these displays during the 60 's and 70 's focused on sensory substitution, where the intention was that tactile stimuli could be used to represent otherwise absent visual or auditory cues to impaired users. To highlight one example from this era, rigorous empirical work on topics ranging from character recognition to the display of patterns representing the spatial aspects of visual scenes were conducted using various versions of TVSS [9], a system most typically incarnated as a 20 by 20 back mounted array of vibrating elements (or tactors). Beyond research with this laudable aim, more recent attention has begun to focus on the potential of vibrotactile stimuli in mobile computing scenarios. This is no doubt due to the dramatic rise of this domain during the last decade, in conjunction with a general recognition

\footnotetext{
*email: ian@whereveriam.org

†email: kym@gist.ac.kr

†email: junhun@gist.ac.kr

**email: ryu@gist.ac.kr
}

that non-visual cues have an important role to play in the interfaces to handheld or wearable computing devices [4], and, when compared to other types of haptic or tactile device, the practical suitability of vibrotactile displays to mobile environments.

Tan [21] provides one of the earliest discussions of vibrotactile displays for mobile computing. Echoing TVSS, the system she describes consists of 9 vibrating elements arranged in a grid and positioned on a user's back. The authors suggest that this device could serve as a saltation display, essentially presenting directional, navigational or orientation information through a 2 dimensional version of the well-known illusion, the cutaneous rabbit. In a later study [20] she validates this technique and suggests this display could be used to provide road directions to drivers. This domain has proven to be popular in subsequent research, with a number of research groups working on this topic. In Holland, van Erp and colleagues [22] have published extensively on the use of vibrotactile displays for navigation, eventually settling on a wrap around torso-mounted display that can be used to directly indicate direction. In tandem with this effort, Lindeman and colleagues [13] have pursued a similar set of objectives, and indeed developed a broadly similar torso based display. In both these cases the tasks that are being considered are high demand - in one paper, van Erp discusses navigation of military speedboats [22], Lindeman a military search task through unfamiliar buildings [13].

Other, more generally applicable topics that have been considered include further work from Tan work on attentional cueing [20] where it has been shown that a spatially localizable vibration delivered to the back can direct attention to a similar visual quadrant. Tan suggests that this could be used to point out potential dangers on the road to drivers, or highlight important onscreen information for workers in safety critical environments such as air traffic control. Work has also focused on human abilities to recognize spatiotemporal patterns, either relying on the well-studied task of letter recognition [24], or using a customized set of stimulus patterns [16]. Research examining how information can be encoded in single vibrating elements has also developed apace. Brewster [4] discusses tactons, the vibratory equivalent of earcons, which are a construct for encoding and displaying structured information through patterns of vibration. Maclean [15] has developed a related concept of hapticons, and a number of authors have begun to examine the emotional and expressive characteristics of vibration [18].

In line with the majority of these authors, our long term interest lies in the area of multi-element wearable tactile displays for general purpose mobile computing: we envisage the use of these displays with mobile phones, PDAs, or other consumer devices. However, as Cholewiak [6] comments, with reference to vibrotactile display systems: "The spatial acuity of the skin has been examined for simple touch but not for the types of vibrating signals used in such devices". Although the body of literature reviewed above is both substantial and informative, due to the fact that vibratory stimuli are not subject to the same perceptual limits as pressure stimuli, basic psychophysical questions relating to the optimal positioning and arrangement of, and stimulus design for, groups of vibrating tactors remains unanswered. Essentially, it is 
currently unclear how the elements in a vibrotactile tactile array might best be arranged on the skin, and it is towards this basic challenge that this paper is addressed.

\section{TACTILE DISPLAYS ON THE ARM}

Our objective is the production of a general purpose body mounted vibrotactile display. Considering such a scenario places restrictions on the design of that display. As Pierce et al. [17] point out in relation to VR hardware, requiring users to don elaborate mechanisms in order to interact with computer systems causes significant problems in and of itself. At the very least users can be reluctant to strap on bulky or restricting devices and this reticence is likely to be exacerbated in the mobile domain. Quite simply, we suggest users will not want to wear large, obtrusive or cumbersome devices. Colloquial evidence for this assertion comes from the transformation of mobile phones from utilitarian black boxes to slick objects of desire, and from recent MP3 players [10] which have been styled more as jewelry than jukebox. Much as with watches and eyeglasses, it appears that more technologically advanced mobile devices are well on their way to making the transition from function to fashion.

Reflecting these sentiments, we suggest that a small and densely populated forearm mounted device (such as that described by Lee et al.[12]) is a likely candidate design; indeed this holds true not just for tactile devices, but for wearable devices in general The forearm is easily accessible to a user, and an established and socially acceptable location upon which to mount a device. Correspondingly, we have focused our investigations on this site.

\section{$2.1 \quad$ Localization}

Several other authors have also considered forearm mounted displays, no doubt in response to these same motivations. Piateski and Jones [16] designed a 3 by 3 array for the under-side of the forearm. Using this device, they conducted an empirical study of the ability to recognize simple patterns including the activation of lines of tactors moving either along or across the arm. They record recognition rates of between $80 \%$ and $96 \%$ for these stimuli, and comment that it appears to be easier to recognize movements across than along the arm, possibly due to the fact that the edges of the arm may serve as landmarks for localizing cues. They contrast this performance with the perfect recognition rates they achieved using similar stimuli displayed on a 4 by 4 back mounted array, and conclude the back may be a more suitable body site on which to mount a vibrotactile display.

As part of a more substantial body of work, Cholewiak and colleagues have studied vibrotactile perception on the arm. In one paper they quantified the parameters required to produce a saltation illusion on a number of body sites including the forearm [8] and in another provided an account of the ability to localize vibrotactile stimuli using a 7 element uni-dimensional array mounted along the underside of the arm [7]. They have also rigorously examined localization performance with torso mounted arrays in a number of different arrangements $[5,6]$.

Taken as a whole, this work provides key information for the design of vibrotactile displays. First and foremost, for our purposes, they report localization rates for vibrotactile stimuli on the forearm. With stimuli spaced $2.5 \mathrm{~cm}$ apart, they report average rates of $46 \%$, with stimuli separated by $5 \mathrm{~cm}$ this increased to $66 \%$. They also discuss the importance of bodily landmarks, in the form of joints, and highlight higher localization rates (of up to $80 \%$ ) at and around these areas. These data strongly suggest that bodily landmarks should be considered in the design of vibrotactile devices. However, the low localization rates they report eventually lead them to conclude that the forearm is an unsuitable site for deployment of an array if absolute localization is required. More positively, in experimentation to assess the effects of altering the frequency and amplitude of vibrotactile stimuli on localization performance, they conclude these parameters have little to no effect. This suggests that the inexpensive, and often non-uniform, tactors used in consumer electronic goods may well make suitable array elements.

Summarizing this literature, it casts a doubtful light on the suitability of the arm as a site for vibrotactile display devices. However, it does not tell the whole story. Regarding localization, the available literature does not approach a complete or systematic account. Specifically, we feel that the role of bodily landmarks, particularly with respect to Piateski and Jones's [16] observation that the edges of the forearm may serve as one such marker, has been inadequately explored. Correspondingly, in section 3.2 we describe a localization study designed to investigate this topic.

\subsection{Intensity}

Beyond simple localization data, tactile displays can also be used to present information such as abstract patterns, or more generally, moving vectors or spatiotemporally rendered lines. By stimulating tactors in sequence, it is possible to create recognizable percepts with forms as complex as alphanumeric characters, and indeed, research has shown this to be the most effective way to present such a stimulus set [24]. However, this work has largely focused on relatively sparse arrays, often relying on subjects constructing a representation of the shapes being displayed through simple repeated stimuli localization. An alternative paradigm is the notion of tactile apparent movement [11], an illusion in which cues presented to two body sites at appropriate timing intervals are perceived as the movement of a single point of contact between the two locations. While these techniques can be effective, in the kind of dense array we envisage, where tactors are mounted extremely close to one another, it may be possible to create the illusion of coherent continuously moving points of stimulation, without the restrictions of rendering motion of a particular speed or in certain specific directions.

Borst and Austay [3] provides one of the few accounts related to this topic. This work describes a series of studies using a dense vibrotactile array positioned under the palm, and contrasts a number of techniques by which arbitrary lines can be displayed. Lines of this sort exist in a virtual coordinate space with a resolution greater than that of the tactile array on which they are being displayed. Two of these techniques simply involved the sequential stimulation of individual tactors with a stimulus of fixed intensity. A third relied on the simultaneous activation of multiple tactors, with the intensity of the stimuli on each varying according to the distance between the tactor and virtual point being displayed. The motivation for this approach was drawn from the graphical process of anti-aliasing, or interpolation, which is used to render smooth lines at sub-pixel resolutions on computer screens. Indeed, the algorithms Borst uses to determine the levels of tactor stimulation are directly drawn from the computer graphics literature. Results from these studies tentatively indicate that, at least for the display of individual lines, this interpolation technique represents an effective strategy, and enables the detection of lines at sub-tactor resolution. Taking a further leaf from the graphics literature, Borst also highlights the importance of an intensity function in this procedure. This function forms a parameterized mechanism for adjusting the relationship between the tactor activation level and the distance to the virtual point being displayed. Essentially adjusting the values passed to this function controls the intensity with which intertactor points are rendered on the display; to apply a visual term, 
this equation controls the display's brightness. Borst reports that using different values in this function exerts a strong effect on the stimuli produced. They “... can be set to make arbitrarily short line segments behave like longer ones"

Underpinning this work is the assumption that the perceived intensity of a vibrotactile stimulus is a combination of the displayed intensity and the size of the area of skin to which it is being presented. That rendering a stimulus of a fixed magnitude to a smaller or larger skin area will result in similar changes to its perceived intensity. Such interactions are well documented in the visual modality and indeed have been formalized to such an extent that Ricco's Law mathematically describes the relationship leading to this kind of spatial summation [19]. Using individual tactors ranging in size from $0.005 \mathrm{~cm}^{2}$ to $5.1 \mathrm{~cm}^{2}$ Verrillo [23] demonstrated that this kind of summation also occurs with vibrotactile cues delivered to the skin. However, it is currently unclear whether or not this effect can be replicated using an array of closely packed tactors. If it can, then it should be theoretically possible to create a uniform transition between the activation of one tactor and the activation of an adjacent one by appropriately varying the intensities of the stimuli displayed on both. Borst's manipulation of his intensity function is, in effect, a manipulation of the parameters of this relationship, although a relatively unquantified one. Given this work suggests that interpolation can lead to performance improvements, and the fact that the ability to render arbitrary lines is a desirable feature for any display, we believe this topic warrants further investigation. To this end, section 3.3 describes a study exploring the relationship between the magnitude of a vibrotactile stimulus and the number of closely packed tactors on which it is being displayed.

\section{EXPERIMENTS}

\subsection{Experimental Overview}

\subsubsection{Tactile Display}

Both experiments were conducted using the same tactors, embedded hardware platform and high-level software interface. The design and construction of this entire system was heavily influenced by that described in detail by Borst and Cavanaugh [2], and as such will only be discussed briefly here. The tactors used were pancake style vibrators, of a proprietary design used in Samsung mobile phones. They are $9 \mathrm{~mm}$ in diameter, and $3 \mathrm{~mm}$ in height. As with other simple tactors, they exhibit a significant latency between the application or removal of current and corresponding movements to peak or idle states. There is also some variability in the response of individual tactors. Cholewiak et al's [7] observation that altering frequency or amplitude of vibrotactile cues does not affect the human ability to localize them suggests that, despite these limitations, these tactors may serve as suitable devices for generating stimuli for use in empirical studies. These devices also have the somewhat dubious advantage that they are typical of the kind of hardware that is likely to appear in any real world system or product.

The tactors were driven by a dedicated microprocessor, a 16 $\mathrm{MHz}$ ATMega 128 [1]. This chip provides a total of 56 digital output lines, each of which can be used to drive a single tactor, and an RS232 interface, which can be used to communicate with a host computer. Using this system, we developed a simple controller on the ATMega which switched on and off the digital outputs in response to instructions received from the RS232 link with the host. Software on the host delivered such instructions, as bit patterns, 500 times a second. During development, the largest array we constructed consisted of 7 by 5 tactors, therefore requiring 5 bytes for a complete update. Each update also included a header byte to ensure proper synchronization, so the total throughput per second reached 3000 bytes (24000 bits), comfortably within the bandwidth of the RS232 link. This rapid update rate was used to enable us to encode Pulse Width Modulation (PWM) signals into this stream. With this technique, we were able to precisely vary the amount of time voltage was supplied to each tactor, which in turn varied the magnitude of the vibration being produced, without yielding any unwanted perceptual artifacts, such as low frequency vibrations. PWM is an established technique for the control of DC electrical devices, and takes advantage of the fact that even though, for example, voltage may only be applied to a device for $50 \%$ of the time it is active, if the duration between active and inactive periods is kept to a minimum, then the device simply behaves as if it was being driven at a lower voltage, and outputs a stimuli of reduced magnitude. In our system we defined 9 PWM levels, spread over an 8 update cycle and ranging from all off to all on. This allowed us to produce what we informally classified as 9 perceptually different vibratory stimuli.

The experiments each used a different configuration of tactile array, but both were constructed in a similar manner. The tactors in each array were glued to lightweight cloth, which was in turn attached to Velcro strips ( 3 in the localization study, a single strip in the intensity study). The Velcro was used to bind the arrays to the forearms of participants. As a stimulus site, we selected the upper-side of the arm, rather than the base (or volar) area, which has been studied in the majority of the previous literature $[7,16]$. This was for purely practical reasons: pilot subjects reported some discomfort if they were required to rest their arms palm up for the duration of the experiments, and we found the array location appeared to become skewed if we allowed them to rotate their arms after it was attached. The forearm is a complicatedly jointed and extremely mobile portion of the body, but it is beyond the scope of this paper to consider how different postures might affect perception. In each study, participants placed their palms face down on a desk and the center of the tactile array was positioned as accurately as possible on a point in the center of the forearm and $7.5 \mathrm{~cm}$ back from the base of the thumb.

\subsubsection{Participants}

The studies both featured 8 participants, and in each 3 were female and 5 male, and their mean age was 27. Despite these similarities, no participant appeared in both studies. The participants were students and employees from our institute, and although some had encountered various versions of our equipment before at demonstrations, none had used it for a protracted period of time. During the first study, we also measured the circumference of participant's wrists around the center point of the tactile array. We recorded a minimum of $15.2 \mathrm{~cm}$, a maximum of $19 \mathrm{~cm}$ and a mean of $17.3 \mathrm{~cm}$.

\subsubsection{Procedure}

The studies also shared a similar procedure. Participants were greeted and escorted to an empty office. They read printed instructions, and then had the opportunity to ask questions about their task. They sat in front of a computer screen and keyboard. The appropriate tactile array was then strapped to their left arm, leaving their right arm free to use the keyboard to make responses. A practice session then began, during which an experimenter remained in the room to resolve any problems that might come up. At the end of the practice, the experimenter left the room and the participants donned headphones and for the remainder of the experiment listened to white noise in order to mask any sounds from the equipment. 


\subsubsection{Task}

The goal of this experiment was to determine localization rates for vibrotactile stimuli on the forearm, in light of previous research reporting that tactors positioned along the center of the forearm and spaced at $25 \mathrm{~mm}$ led to an accuracy rate of $44 \%$ [7]. Most importantly, we wanted to contrast performance with stimuli positioned along the forearm, against that achieved with stimuli positioned across the forearm. In order to do so, and to link into this previous work, we constructed a 3 by 3 element array in which the tactors were separated by $25 \mathrm{~mm}$. This device is shown in Figure 1. Needless to say, with a total width approaching $6 \mathrm{~cm}$ and the slightest subject possessing a wrist circumference of just $15.2 \mathrm{~cm}$, this contraption extended to greater or lesser portions of the curved parts of the participant's wrists. Consequently, it is possible that wrist size may have influenced the results of this study.

An English version of the experimental interface is shown in Figure 2, while Figure 3 shows the array positioned on a subjects arm. Each trial consisted of the subjects pressing a key to begin the trial, followed by a $1000 \mathrm{~ms}$ pause followed by vibration being displayed to one of the tactors for $500 \mathrm{~ms}$. Subjects then had to respond by pressing one of the number keys on the numerical keypad (the section typically situated on the right of the main keyboard) to indicate which of the tactors they believed had been activated. This response was recorded. To minimize confusion, the on screen layout of the interface corresponded to the physical layout of the numeric keypad. After a selection was made, visual highlighting revealed both the user's response and the correct answer. If no key press occurred, the trial timed out after 10 seconds and no data was recorded. In both cases, a new trial then commenced. Subjects were instructed that they were able to rest freely in between trials.

During the experimental session, a total of 270 trials were presented to each participant, 30 for each tactor. Trials were displayed in a random order. The practice session involved the same process, but used a reduced stimulus set consisting of 15 trials per tactor.

\subsubsection{Results}

Recognition rates for each of the nine tactors are presented in Figure 4 . As can be seen, they vary substantially, ranging from as low as $22 \%$ to as high as $76 \%$. An ANOVA revealed these differences to be significant $(\mathrm{F}(8,7)=7.217, \mathrm{p}<0.001)$, but the only results that were born out with post-hoc t-tests (incorporating Bonferroni confidence interval adjustments) was that tactor three resulted in significantly better performance than tactors six and nine (respectively $\mathrm{p}<0.05$ and $\mathrm{p}<0.01$ ). Error data for each tactor is shown in Table 1. In order to compare the performance between tactors mounted along the forearm and those mounted across we collapsed these data according to row and column, conflating a portion of the errors with the correctly localized responses. This created two data sets: one representing all results in which participants responded with the correct column of the array, the other in which they responded with the correct row. Means for these two data are shown in Figure 5. A t-test revealed the difference between these values to be significant $(\mathrm{t}=8.535$, $\mathrm{p}<0.001)$.

\subsubsection{Discussion}

Our results fit relatively well with that reported in Cholewiak's study of tactors mounted along the underside of the arm, and

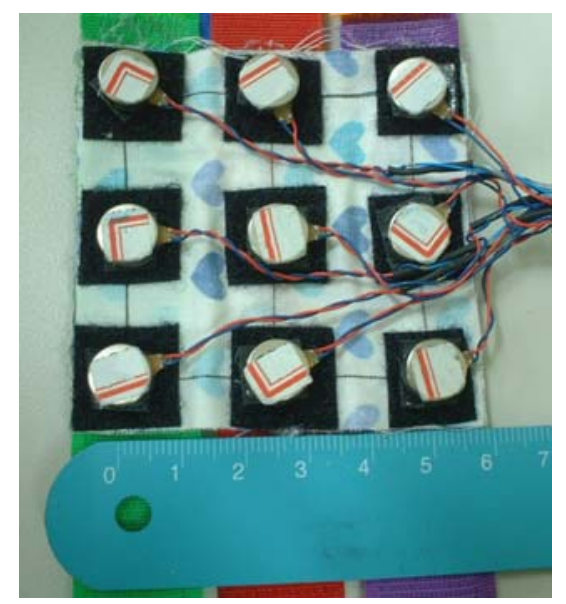

Figure 1: 3 by 3 vibrotactile array used in localization study.

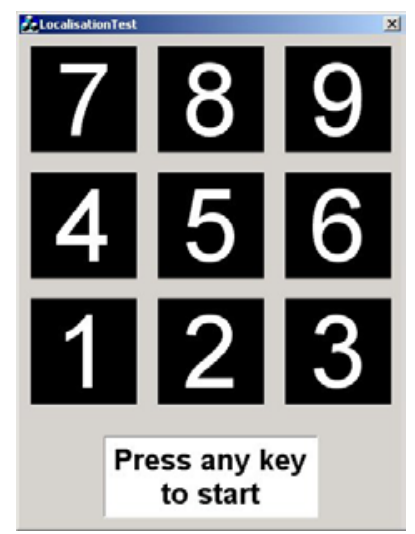

Figure 2: Interface to the localization study.

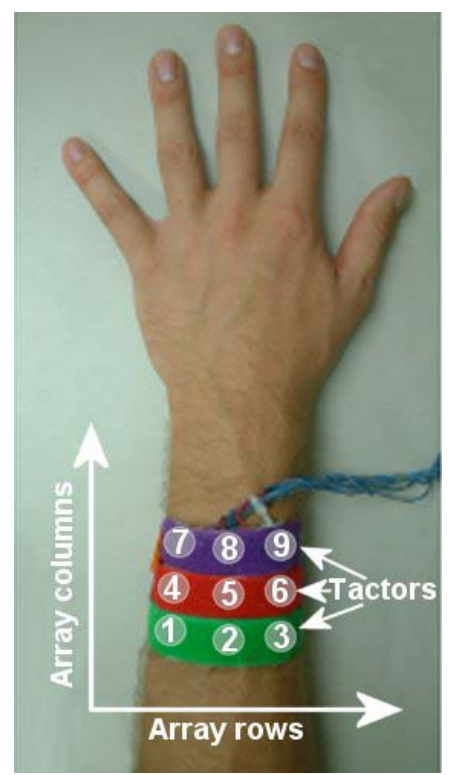

Figure 3: A participant wearing the 9 tactor array used in the localization study. Not to scale image overlays highlight details of tactor arrangement. 
separated by $25 \mathrm{~mm}$ [7]. This work reports a mean localization rate of $46 \%$, which is comparable to the rate of $53 \%$ observed in this study when considering tactors spread along the length of the forearm. The differences between these figures may be due to the different stimulus site used (the top rather than base of the forearm), but is more likely to be attributable to the fact that Cholewiak considers a 7 item array, and it has been observed that the edges of a vibrotactile display afford increased localization rates when compared to interior items [5]. Regardless, it would appear that there is little difference in sensitivity between sites on the top or base of the forearm for tasks involving the localization of vibrotactile stimuli. This is in itself a useful observation for the designers of vibrotactile displays.

The results revealing the difference in performance between localizing vibration to the correct rows or columns of the array are more dramatic. From these figures the conclusion that an array of three tactors mounted across the forearm, in line with such common accouterments as the strap of a watch, affords a much greater localization rate than one mounted perpendicularly to this, along the length of the arm, seems inarguable. It is likely that this is due to the fact that, as Piateski and Jones [16] observed, the sides of the arm serve as bodily landmarks or reference points with which stimuli can be associated. Furthermore, it is possible that these localization rates could be further improved in a simple 3 tactor system; with fewer tactors, it seems likely that the stimuli they produce would be easier for a subject to identify and learn.

Considering the results relating to the individual tactors, it is also worth noting that the only significant differences occur in the three tactors situated on the right-hand side of the array (tactors 3 , 6 and 9), which was positioned on the "thumb-side" of the top surface of the forearm (see Figure 3 for a graphical depiction). It appears that localization of the position of a vibrotactile stimulus along this portion of the forearm is more challenging than at other, adjacent, locations. From this experiment it is unclear why this is, and this may warrant a more detailed investigation.

\subsection{Experiment 2 - Intensity}

\subsubsection{Task}

This experiment was designed to investigate the effect of varying the magnitude of a vibrotactile stimulus against the effect of varying the size of the area of the skin to which it is being displayed. In order to do this a simple three tactor display system was used. Each tactor was mounted close to the remaining pair in a triangular arrangement, as illustrated in Figure 6. As the tactors were attached to a fabric wristband, some distance was left around each one in an attempt to eliminate the uncontrollable impacts they might make against one another if placed immediately adjacent. The distance between tactor centers was approximately $15 \mathrm{~mm}$, under the localization limits found in the previous study, and consequently, very likely to be significantly under the twopoint threshold for vibration [19]. This tactor configuration enabled us to vary the amplitude of the displayed vibrations through the PWM capability of our controller and, crudely, the area of stimulation by simply activating a variable number of tactors. To keep this manipulation hidden from subjects, the array was left face down during the set up phase of the experiment.

Quantifying the relationships between two stimuli parameters is a complex task, and the intention of this initial investigation was more to demonstrate the presence or absence of an interaction between the parameters of intensity and site size than to provide a full account of their relationship. Correspondingly we adopted a simple experimental model using a modified forced choice response paradigm in which participants compared two stimuli

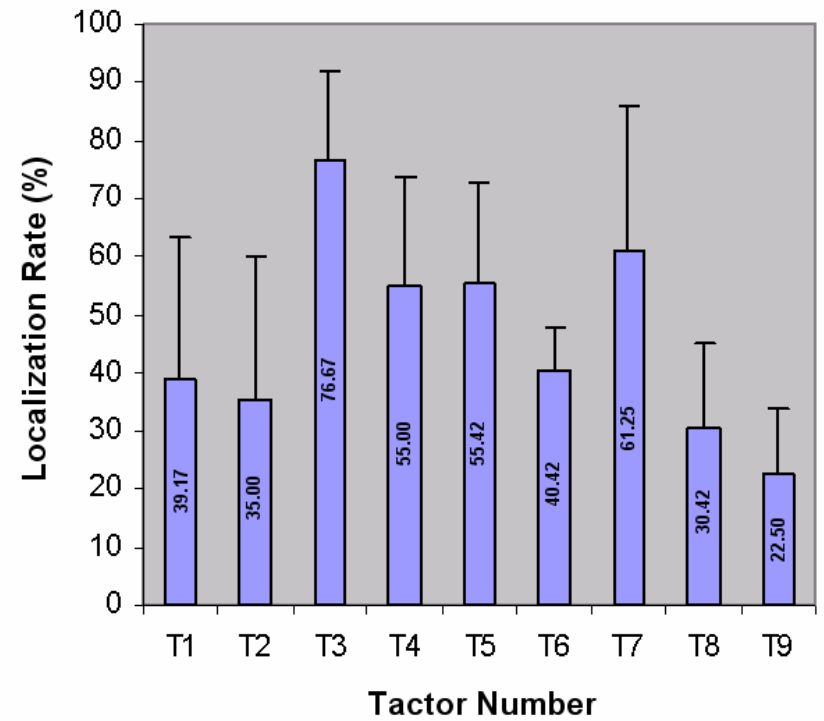

Figure 4: Percentage localization rates by tactor (bars show standard dev). Tactor location by number shown in Figure 3.

\begin{tabular}{|c|c|c|c|c|c|c|c|c|}
\hline \multicolumn{3}{|c|}{ Tactor 7} & \multicolumn{3}{|c|}{ Tactor 8} & \multicolumn{3}{|c|}{ Tactor 9} \\
\hline 61.3 & 2.5 & 0.4 & 12.5 & 30.4 & 3.3 & 0 & 3.3 & 22.5 \\
\hline 25 & 3.8 & 1.3 & 7.9 & 40.4 & 2.9 & 0 & 8.3 & 42.5 \\
\hline 5.4 & 0 & 0.4 & 0.4 & 1.7 & 0.4 & 0.4 & 0.8 & 22.1 \\
\hline \multicolumn{3}{|c|}{ Tactor 4} & \multicolumn{3}{|c|}{ Tactor 5} & \multicolumn{3}{|c|}{ Tactor 6} \\
\hline 28.8 & 2.1 & 0.8 & 3.8 & 0.4 & 2.1 & 0 & 0.4 & 5.8 \\
\hline 55 & 3.3 & 0.4 & 8.8 & 55.4 & 6.3 & 0 & 5.8 & 40.4 \\
\hline 9.2 & 0 & 0.4 & 2.1 & 9.6 & 0.4 & 0 & 6.3 & 41.3 \\
\hline \multicolumn{3}{|c|}{ Tactor 1} & \multicolumn{3}{|c|}{ Tactor 2} & \multicolumn{3}{|c|}{ Tactor 3} \\
\hline 13.3 & 0.8 & 0 & 2.1 & 5 & 1.7 & 0 & 0 & 1.3 \\
\hline 37.9 & 5.4 & 0.8 & 7.9 & 30 & 7.1 & 0 & 0.4 & 12.9 \\
\hline 39.2 & 1.7 & 0.8 & 5.8 & 35 & 5.4 & 0.8 & 7.9 & 77.6 \\
\hline
\end{tabular}

Table 1: Distribution of responses in the localization study, expressed as mean percentage values. Bold figures indicate correctly localized responses.

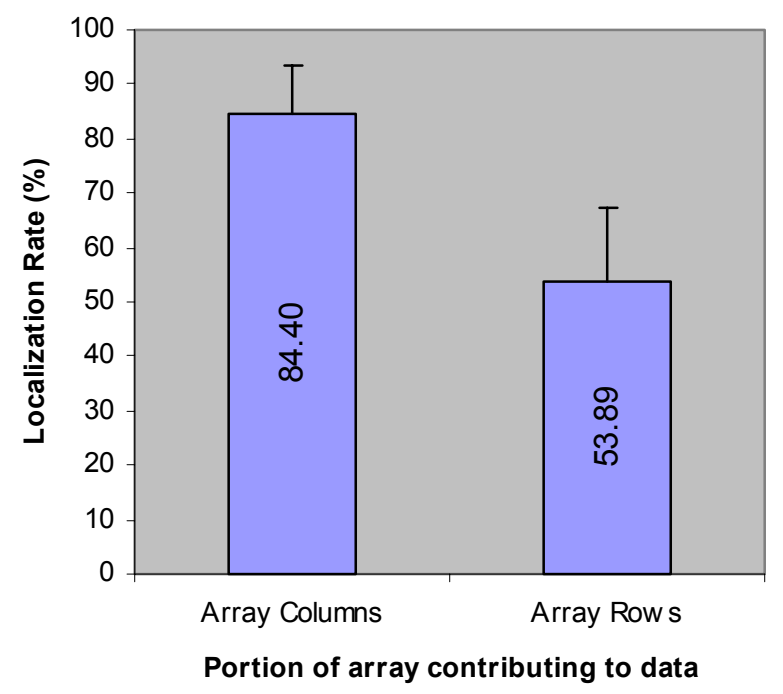

Figure 5: Percentage localization rates by array column and row (bars show standard deviation) 


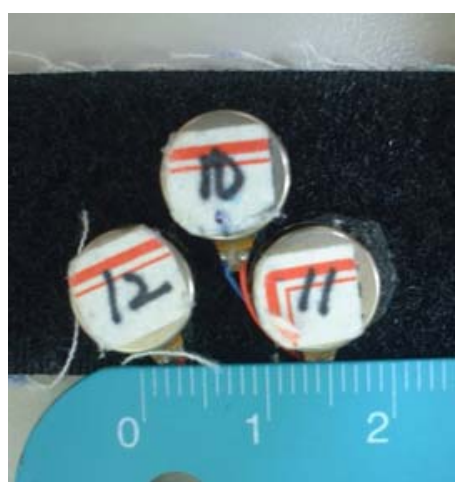

Figure 6: 3 element vibrotactile array used in intensity study.

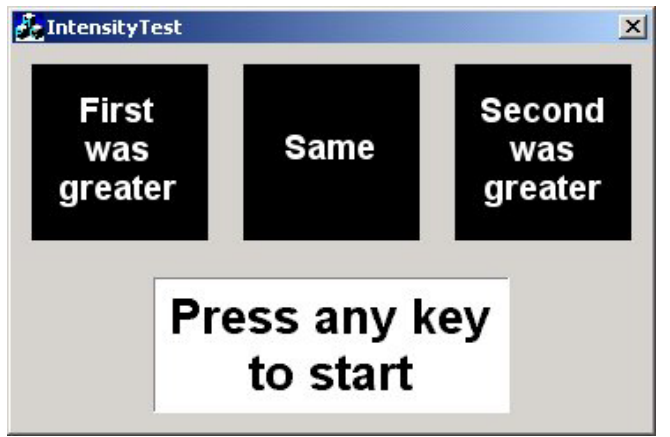

Figure 7: Interface to the localization study.

and judged either one or the other to be of greater magnitude (or intensity), or the pair to be of the same magnitude. The terms magnitude and intensity were not explicitly defined for participants; they were largely left to interpret them independently.

The stimulus set for this study consisted of the highest six intensities that could be produced using the system's PWM interface, displayed on between one and three tactors. If more than one tactor was being used to display a stimulus, each was always driven with the same PWM pattern. This led to a total of 18 possible stimuli. Each stimulus was compared against the full set, leading to 18 by 18 trials: 324 in total. The decision to not use the full range of PWM values was reached because we were uninterested in examining values that may be approaching threshold, and due to the fact that observations of pilot subjects revealed that at 324 trials, the study was already lengthy.

Figure 7 shows an English version of the interface to this study. As with the localization study, each trial began with the participants tapping a key on a computer keyboard. After a 1000 ms pause, a vibration was displayed for $500 \mathrm{~ms}$, followed by another $1000 \mathrm{~ms}$ pause, followed by a second $500 \mathrm{~ms}$ of vibration. Participant's then used either the left, down or right arrow keys on a computer keyboard to indicate whether the first stimuli was greater than the second, that they were the identical or that the second was greater than the first. This spatial layout of keys matched that of the graphics of the on-screen interface, and participants were asked to leave their hands on these three keys for the duration of the experiment. They received feedback indicating which response they made, but not regarding its correctness. If participants did not respond within 10 seconds, a timeout occurred and no data was recorded. Either way, a new trial began at this point. Trials were presented in a random order, and a practice session consisting of 54 randomly selected trials took place immediately before the experimental session.

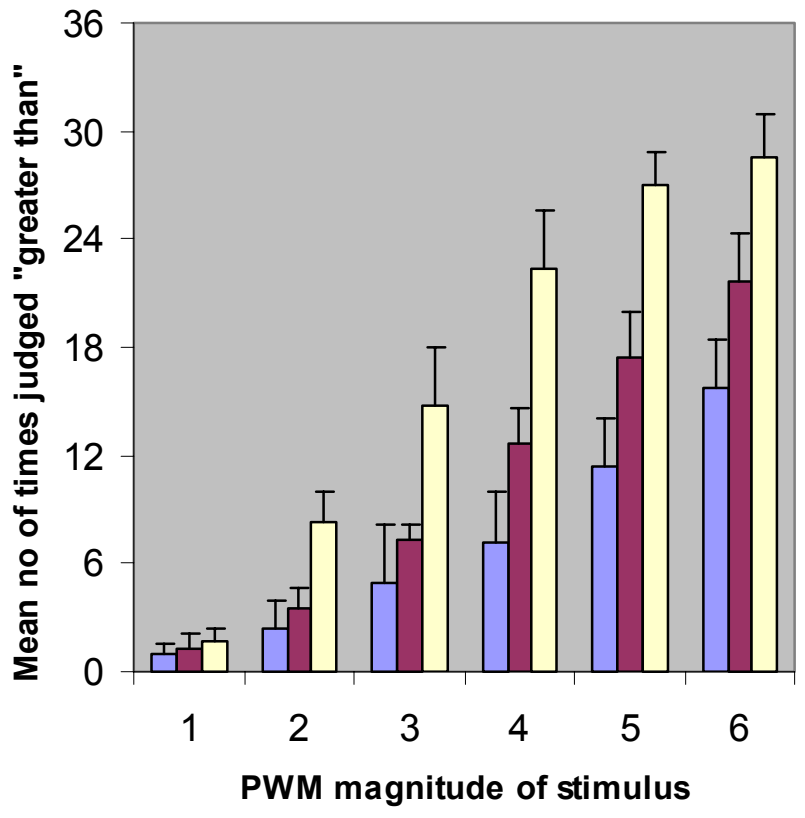

1 Tactor $\square 2$ Tactor $\square 3$ Tactor

Figure 8: Effect of PWM magnitude and number of tactors on perceived stimulus intensity (bars show standard dev)

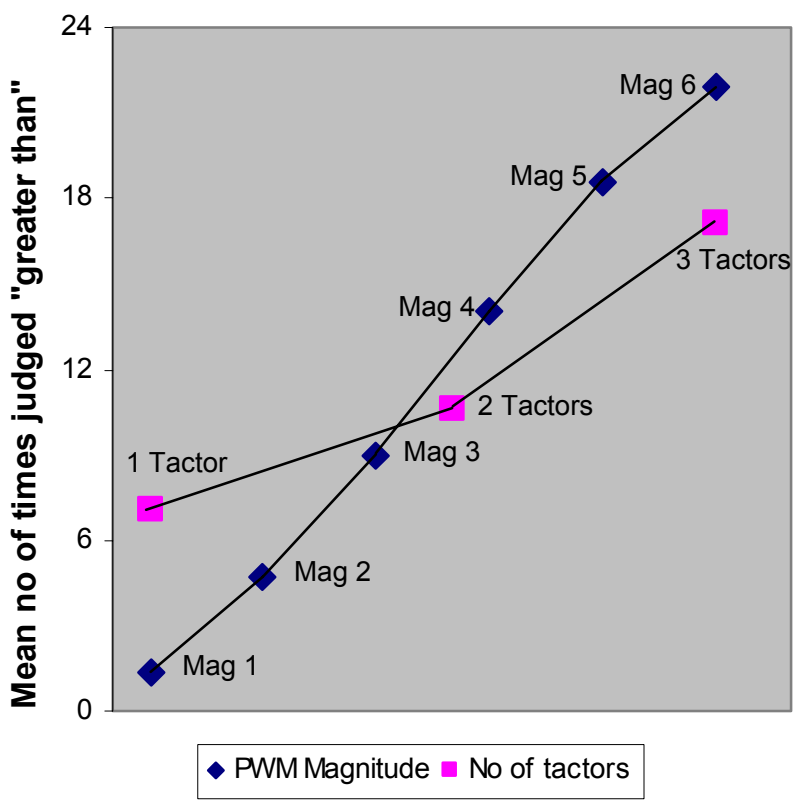

Figure 9: Interaction between the PWM magnitude of a stimulus and the number of tactors it is displayed on.

\subsubsection{Results}

The data took the form of pair wise judgments contrasting one stimulus against another. The simplest way to process and represent these data is to simply tally the number of times a given stimulus was rated as being greater than another. As perceived intensity increases, this count should similarly increase. These data are shown in Figure 8. A 2-way ANOVA revealed that both 
the PWM intensity of a stimulus and the number of tactors on which it was displayed exerted a significant effect on this figure (respectively $\mathrm{F}(5,7)=300.442, \mathrm{p}<0.001$ and $\mathrm{F}(2,7)=241.210$, $\mathrm{p}<0.001)$. A significant interaction between these parameters was also uncovered $(\mathrm{F}(5,2)=13.650, \mathrm{p}<0.001)$. Figure 9 plots this interaction. Post-hoc t-tests, incorporating Bonferroni confidence interval adjustments, revealed significance differences between each value of the both of the stimulus parameters (all at $\mathrm{p}<0.001$ ).

\subsubsection{Discussion}

This experiment clearly reveals that the stimulation of multiple, closely sited tactors interacts with the magnitude of the vibration being presented (as determined by the level of electrical power being delivered) to create a single percept of perceived intensity. All values of both the independent variables in this study significantly affected the number of times a given stimulus was rated to be the greater of a pair. This result comes as little surprise for the intensity parameter, but determining that by adjusting the stimulus site size it is possible to influence perceived intensity constitutes an important step. Theoretically, this result suggests that it may be possible to create a mapping between two closely sited tactors whereby their intensities can be interactively adjusted from a state in which only one is activated to a state where this activation has shifted to the other without altering the perceived intensity of the displayed vibration during this transition.

The implications of this for the design of dense vibrotactile displays are positive. By exceeding the threshold for the localization of vibration, it may be possible to create a display that is capable of rendering arbitrarily moving stimuli that cross the skin with the sensitivity and resolution with which the touch of a fingertip can be felt. Some evidence that the amount of quantifiable information that can be transmitted by a display with such a capability already exists [2], but even in its absence there remains considerable attraction in the ability to render such richer stimuli. With respect to interactive systems, abstract notions such as quality are beginning to be recognized as important factors in the construction of interfaces and the ability to produce output that is richer and more pleasing is likely to contribute to this. To rephrase this using more traditional terminology, it may allow the production of cues that simply feel more realistic.

However, this study does not provide the kind of detailed perceptual scaling that would allow the formalization of the relationship between stimulus site size and displayed intensity. Further research is required determine both explicit links between particular parameter values, and to uncover where those links may break down. For example, although from the results reported here, it appears that it should be possible to create the kinds of seamless moving points discussed in the previous paragraph, this has not been empirically determined. Although it is clearly possible to use stimulus size to modulate perceived intensity, in reality it is unlikely that this can be achieved without the production of some perceptual artifacts. Much like a visually anti-aliased point appears blurred, dividing the energy in a vibrotactile stimulus from its original incarnation on a single tactor to a new version displayed on two or more is likely to result in a percept that feels similar, rather than identical, to the original.

\section{General Discussion And Conclusions}

Forearm mounted vibrotactile displays have recently been examined by a number of authors. The idea of mounting a display on the forearm is an appealing one. Perhaps of all possible body sites, it is the most attractive for such a purpose as it is both physically and visually accessible to users, and is an easy, established and acceptable part of the body on which to wear an electronic device. However, recent research comparing the performance achieved on this site against that found on the torso has not been supportive of its use. Both Cholewiak et al. [7] and Piateski and Jones [16], respectively conducting localization and pattern recognition tasks, conclude that performance levels are not high enough to justify the development of a forearm mounted device. Piateski and Jones directly compare performance on the forearm against that on the torso and find the torso to be superior. However, in contrast to this work, we believe the studies described in this paper argue strongly in favor of the forearm as a site for the display of vibrotactile cues.

The first study reported, investigating localization, shows that substantial increases in performance can be achieved through taking advantage of spatial layouts that maximize the use of bodily landmarks, such as the edges of the arm. The data suggest that through the simple act of using an array that is mounted across the forearm, in line with the strap of a watch, instead of along the forearm, localization rates can be improved by over $30 \%$, rising to a level which rivals that reported in studies of localization in belt like arrays around the torso [6]. Without the additional complexity of the two-dimensional system used here, we suggest a simple uni-dimensional array mounted across the wrist could result in localization rates that are still greater. The effects of lengthier practice are also likely to boost performance [6]. Taking altogether, these facts and assertions are compelling; they combine to strongly suggest that such an array would act as a useful and reliable output device.

Needless to say, a further practical advantage to such a laterally arranged array is that it is natively smaller and easier to wear than one mounted along the arm. Using a single wrist strap, it is possible to position three or more tactors. If sites on both the top and bottom of the forearm are employed this study suggests that as many as six tactors could be attached to a single band, and good localization performance be maintained. In fact, assessing user performance with such a display represents an interesting avenue for a follow-up localization study. Such a study would also serve to verify the notion that a uni-dimensional array would lead to higher performance than that recorded with the two dimensional array examined here.

The second experiment, investigating the interaction between stimulus site size and stimulus intensity, examines the topic of spatial summation. It asks the question of whether the simultaneous presentation of vibrotactile stimuli which are positioned sufficiently close together that they are beneath both localization and two-point threshold limits (which tend to much greater than localization limits [14]) are instead perceived as a single stimulus with a magnitude greater than that of its component elements. Although it does not formalize the relationship between these two parameters, the study does provide strong evidence that spatial summation does occur with vibrotactile stimuli on this sort. It reports that both parameters exhibited significant upwards trends, and significantly interacted with one another. Further experiments to more formally establish the properties of spatial summation for vibrotactile stimuli are an obvious next step for this work.

However, in tandem with this it is worth noting that there is currently very little work suggesting how spatial summation might be put to use in the design of vibrotactile cues for information display. Borst and Austay's [2] recent work represents the most significant exception to this absence, and in line with their results, we speculate that it may be possible to use the technique to render a much richer set of compound, dynamically moving stimuli than is possible using the tactile apparent movement [11] and the cutaneous rabbit illusions [20] that have been the focus of 
previous attempts to achieve this. We believe that it would be interesting to construct a relatively large scale, but extremely dense wrist mounted vibrotactile array, in order to further investigate the properties of the information and dynamic stimuli that it can effectively display. We believe that an output device with the capabilities to render substantially richer stimuli than the majority of those currently being considered would lend itself to a much wider set of application areas, and that pursuing these represents another interesting avenue for future investigations.

To conclude, the overall goal of this work was to investigate the feasibility of forearm mounted vibrotactile arrays in light of recent studies suggesting they may be ineffective. We attempted to examine this topic from a fresh perspective and, as Cholewiak \& Beede [5] put it "push the envelope" of tactor density, and vibrotactile display paradigms. We believe that the joint reports of substantially increased localization rates, and (due to the fact that the results showing summation demonstrate that vibrotactile perception is more than just localization) an empirical justification for constructing dense arrays, we have achieved this objective. Future work can build on the fundamentals reported here, not only extending the psychophysical data, but also directly exploring new applications and user scenarios.

\section{ACKNOWLEDGEMENTS}

This work was supported by South Korean Ministry of Information and Communication through the Realistic Broadcasting IT Research Center (RBRC) and the Next Generation PC Project.

\section{REFERENCES}

[1] Atmel, "Atmel ATMega128". 2005, http://www.atmel.com/dyn/ products/product_card.asp?part_id=2018

[2] Borst, C.W. \& A.V. Austay. "Bi-level and Anti-aliased Rendering Methods for a Low-Resolution 2D Vibrotactile Array". in Proceedings of WorldHaptics, 2005, Pisa, Italy.

[3] Borst, C.W. \& C.D. Cavanaugh, "Haptic Controller Deisgn and Palm Sized Vibrotactile Array". 2004, Center for Advanced Computer Studies, University of Louisiana at Lafayette.

[4] Brewster, S. \& L. Brown. "Tactons: Structured Tactile Messages for Non-Visual Information Display". in Proceedings of Australasian User Interface Conference, 2004, Dunedin, NZ, Australian Computer Society.

[5] Cholewiak, R.W. \& K. Beede. "The Representation of Space Through Static and Dynamic Tactile Displays". in Proceedings of HCI International 2005, 2005, Las Vegas, NV.

[6] Cholewiak, R.W., J.C. Brill, \& A. Schwab, "Vibrotactile localization on the abdomen: Effects of place and space", Perception \& Psychophysics, 2004, 66(6): pp. 970-987.

[7] Cholewiak, R.W. \& A.A. Collins, "Vibrotactile localization on the arm: Effects of place, space and age", Perception \& Psychophysics, 2003, 65(7): pp. 1058-1077.

[8] Cholewiak, R.W., A.A. Collins, \& J.C. Brill. "Spatial factors in vibrotactile pattern perception". in Proceedings of EuroHaptics'01, 2001, Birmingham, UK.

[9] Collins, C.C., "Tactile television - Mechanical and electrical image projection", IEEE Transactions on Man-Machine Systems, 1970(11): pp. 65-71.

[10] iRiver, "iRiver N10". 2005, http://www.iriver.com/html/company/ press/copr_pr_view.asp?idx $=79$
[11] Kirman, J.H., "Tactile apparent movement: The effects of interstimulus onset interval and stimulus duration", Perception \& Psychophysics, 1974, 15(1): pp. 1-6.

[12] Lee, B., Lee, J., Cha, J., Seo, C. \& Ryu, J., "Immersive Live Sports Experience with Vibrotactile Sensation". in Proceedings of Interact'05, 2005, Rome, Italy.

[13] Lindeman, R.W., et al. "Effectiveness of Directional Vibrotactile Cueing on a Building Clearing Task". in Proceedings of CHI'05, 2005, Portland, OR, ACM Press.

[14] Loomis, J.M., "An investigation of tactile hyperacuity", Sensory Processes, 1979, 3: pp. 289-302.

[15] Maclean, K. \& M. Enriquez. "Perceptual Design of Haptic Icons". in Proceedings of EuroHaptics'03, 2003, Dublin, Ireland.

[16] Piateski, E. \& L. Jones. "Vibrotactile Pattern Recognition on the Arm and Torso". in Proceedings of WorldHaptics, 2005, Pisa, Italy, IEEE.

[17] Pierce, J.S., Pausch, R., Sturgill, C.B. \& Christiansen, K.D, "Designing A Successful HMD-Based Experience", Presence, 1999, 8(4): pp. 469-473.

[18] Rovers, L. \& H. van Essen. "Design and evaluation of Hapticons for enriched Instant Messaging". in Proceedings of EuroHaptics'04, 2004, Munich, Germany.

[19] Sekuler, R. \& R. Blake, "Perception". 2002: Mcgraw-Hill College.

[20] Tan, H.Z., et al., "A Haptic Back Display for Attentional and Directional Cueing", Haptics-e, 2003, 3(1).

[21] Tan, H.Z. \& A. Pentland, "Tactual Displays for Wearable Computing", Personal Technologies, 1997, 1: pp. 225-230.

[22] van Erp, J.B.F., et al. "Vibrotactile Waypoint Navigation at Sea and in the Air: two Case Studies". in Proceedings of Eurohaptics'04, 2004, Munich, Germany.

[23] Verrillo, R.T., "Effect of Contactor Area on the Vibrotactile Threshold", Journal of the Acostical Society of America, 1963, 35(12): pp 1962-1996.

[24] Yanagida, Y., Kakita, M., Lindeman, R., Kume, Y. \& Tetsutani, N., "Vibrotactile Letter Reading Using a Low-Resolution Tactor Array". in Proceedings of The Twelveth Haptic Symposium on Haptic Interfaces for Virtual Environment and Teleoperator Systems, 2004, Chicago, USA, IEEE. 